\title{
Evidence for paternal transmission and heteroplasmy in the mitochondrial genome of Silene vulgaris, a gynodioecious plant
}

\author{
DE McCauley, MF Bailey, NA Sherman and MZ Darnell
Department of Biological Sciences, Vanderbilt University, Nashville, TN 37235, USA \\ DE McCauley, MF Bailey, NA Sherman and MZ Darnell
Department of Biological Sciences, Vanderbilt University, Nashville, TN 37235, USA
}

\begin{abstract}
Gynodioecy refers to the co-occurrence of females and hermaphrodites in the same population. In many gynodioecious plants, sex is determined by an epistatic interaction between mitochondrial and nuclear genes, resulting in intragenomic evolutionary conflict, should the mitochondrial genome be maternally inherited. While maternal inheritance of the mitochondrial genome is common in angiosperms, few gynodioecious species have been studied. Here, the inheritance of the mitochondrial genes atp $A$ and $\operatorname{coxl}$ was studied in 318 Silene vulgaris individuals distributed among 23 crosses. While maternal inheritance was indicated in $96 \%$ of the individuals studied, one or more individuals from each of four sib groups displayed a genotype that was identical to the father, or that did not match either parent. Given evidence that inheritance is not strictly maternal, it was hypothesized that some individuals could carry a mixture of
\end{abstract}

Keywords: mitochondrial DNA; heteroplasmy; paternal transmission; recombination; gynodioecy; Silene

maternally and paternally derived copies of the mitochondrial genome, a condition known as heteroplasmy. Since heteroplasmy might be difficult to detect should multiple versions of the mitochondrial genome co-occur in highly unequal copy number, a method was devised to amplify low-copy number forms of atpA differentially. Evidence for heteroplasmy was found in 23 of the 99 individuals studied, including cases in which the otherwise cryptic form of atpA matched the paternal genotype. The distribution of shared nucleotide sequence polymorphism among atp $A$ haplotypes and the results of a population survey of the joint distribution of atp $A$ and $\operatorname{cox} I$ haplotypes across individuals supports the hypothesis that heteroplasmy facilitates formation of novel mitochondrial genotypes by recombination.

Heredity (2005) 95, 50-58. doi:10.1038/sj.hdy.6800676

Published online 13 April 2005

\section{Introduction}

Maternal inheritance of the mitochondrial genome has been shown in most species of angiosperms that have been studied, and thus the fitness of mitochondrial genes is usually considered to be a function of seed production (Reboud and Zeyl, 1994; Mogensen, 1996). However, maternal transmission of the mitochondrial genome in angiosperms is not universal, with paternal or biparental cytoplasmic inheritance having been observed in a few species (Nagata et al, 1999). Since the inheritance of the mitochondrial genome has only been studied in a small fraction of all wild species of angiosperms (Zhang et al, 2003), it is not clear how often nonmaternal inheritance might be a feature of the genetics of natural plant populations or influence the fitness of mitochondrial genes.

One consequence of biparental inheritance is that transmission of copies of mitochondrial genes through both pollen and ovules could result in seeds that carry a heterogeneous collection of mitochondrial genomes inherited from both the mother and the father. That is, biparental inheritance could lead to heteroplasmy. In such cases, intraindividual genetic drift during organel-

Correspondence: DE McCauley, Department of Biological Sciences, Vanderbilt University, PO Box 351634, Nashville, TN 37235, USA.

E-mail: david.e.mccauley@vanderbilt.edu

Received 24 September 2004; accepted 28 February 2005; published online 13 April 2005 lar replication, cell division, and growth (Birky, 2001; Rand, 2001) could result in very unequal representation of different copies of mitochondrial genes in different modules of the plant or among the offspring of a heteroplasmic parent, greatly complicating the interpretation of crossing studies. Further, heteroplasmy could facilitate the creation of novel mitochondrial recombinants (Saville et al, 1998; Städler and Delph, 2002). The extent of mitochondrial heteroplasmy in natural populations of plants, and its biological significance, also remains largely unexplored. Further studies of mitochondrial DNA (mtDNA) inheritance and heteroplasmy, encompassing a wide diversity of plant taxa and life histories, would seem warranted.

Knowledge of the inheritance of the mitochondrial genome would seem particularly relevant in gynodioecious plants. Gynodioecious species are those in which hermaphroditic and female individuals co-occur in the same population. In gynodioecious systems, sex determination is often under cyto-nuclear genetic control, resulting from an epistatic interaction between cytoplasmic male sterility (CMS) elements found in the mitochondrial genome and nuclear loci that restore male fertility (Saumitou-Laprade et al, 1994; Budar et al, 2003). CMS-based gynodioecy has been cited as one of the most widely studied examples of intragenomic conflict, owing to the difference between CMS elements and nuclear restorer loci in mode of transmission, and in the currency of fitness (eg Cosmides and Tooby, 1981; Hurst et al, 1996; 
Werren and Beukeboom, 1998). A pleiotropic effect of male sterility is often an increase in the quantity or quality of seeds produced by females, compared to hermaphrodites, resulting in a higher cytoplasmic fitness for those individuals carrying unrestored CMS elements (Frank, 1989; Couvet et al, 1990; Budar et al, 2003).

Strict maternal inheritance of the mitochondrial genome is assumed in most mathematical models of sex ratio evolution in gynodioecious systems with cytonuclear sex determination (Jacobs and Wade, 2003). With paternal or biparental inheritance, the high seed production of females can occasionally contribute to the cytoplasmic fitness of their mates. Occasional transmission of the mitochondrial genome through pollen also raises the possibility that some individuals could be heteroplasmic for two or more forms of CMS, each requiring different mechanisms of restoration.

Despite its significance for the evolution and maintenance of gynodioecy, our knowledge of patterns of inheritance of the mitochondrial genome in gynodioecious species is limited. Similarly, few studies of gynodioecious species have been designed to detect mitochondrial heteroplasmy or its consequences. Here, we report evidence that the inheritance of the mitochondrial genome in Silene vulgaris, a gynodioecious plant, is not strictly maternal, that heteroplasmy may be a common feature of this species, and that one population-level consequence of this heteroplasmy is a reduction of gametic disequilibrium among alleles at two mitochondrial loci. While the results presented here are based on properties of the atpA and coxI mitochondrial loci, genes not known to be involved in CMS in $S$. vulgaris, it is hoped that the information learned from the study of these two genes will reflect more general properties of the $S$. vulgaris mitochondrial genome, including those specific regions of the genome responsible for CMS that are yet to be identified.

\section{Materials and methods}

\section{Study system}

S. vulgaris is a weedy gynodioecious species native to Europe and introduced to North America over 250 years ago. It has been the subject of numerous studies designed to investigate the interaction between its gynodioecious breeding system and other features of its population biology (eg Jolls and Chenier, 1989; Pettersson, 1992; McCauley, 1998; McCauley and Brock, 1998; McCauley et al, 2000; Ingvarsson and Taylor, 2002; Emery and McCauley, 2002; Olson and McCauley, 2002). Previous crossing studies have indicated that genetic control of sex expression is cytonuclear, with evidence that multiple forms of CMS can be found in natural populations and that these CMS factors interact epistatically with multiple nuclear restorer loci (Charlesworth and Laporte, 1998; Taylor et al, 2001). Indirect evidence for occasional heteroplasmy of CMS elements also exists (Andersson, 1999), based on studies of gynomonoecious plants (ie those in which female and hermaphrodite flowers cooccur on the same individual).

\section{Genetic markers}

The investigations of mitochondrial variation at the individual, family, and population level reported here all make use of restriction fragment length polymorphism (RFLP) obtained by digesting the amplification products of polymerase chain reactions (PCR) with the appropriate restriction enzymes. In order to develop PCR/RFLP mtDNA markers portions of two mitochondrial genes, atpA and coxI, were each amplified from a number of $S$. vulgaris individuals using primers developed by CW dePamphilis of Pennsylvania State University (Barkman et al, 2000; Bowe et al, 2000). Observed differences between individual $\operatorname{atp} A$ sequences included the gain or loss of several $A l u \mathrm{I}$ restriction sites and several MspI restriction sites. All of the sequenced individuals could be placed into one of six categories (referred to as haplotypes $\mathrm{A} \rightarrow \mathrm{F}$ ) based on the presence or absence of $A l u \mathrm{I}$ and $M s p \mathrm{I}$ restriction sites within the $1083 \mathrm{bp}$ region of atpA amplified by the PCR. This information was used to develop a PCR/RFLP-based protocol for the rapid genotyping of individuals used in this study. For each individual, DNA was extracted from a single leaf, or two paired leaves from the same branch, using either Qiagen Dneasy plant DNA extraction kits or CTAB extraction buffer (Doyle and Doyle, 1987). This genomic DNA was used as a template for PCR using the atp $A$ primers mentioned above. A $10 \mu \mathrm{l}$ aliquot of the PCR product was digested with $A l u \mathrm{I}$, and the resulting fragments visualized by electrophoresis in a $4 \%$ Metaphor agarose gel stained with ethidium bromide. A separate $10 \mu \mathrm{l}$ aliquot of the PCR product was digested with $M s p I$, with the digestion products again visualized by electrophoresis and ethidium bromide staining. The $A l u \mathrm{I}$ and MspI fragment profiles obtained for each individual determined which of the six atpA PCR/RFLP haplotypes it carried. A similar protocol was developed for determining coxI haplotypes, except that the restriction profile was based on double digestion of a single $10 \mu \mathrm{l}$ aliquot of the $1437 \mathrm{bp}$ coxI PCR product with MspI and $D d e I$. Three coxI haplotypes could be detected by this method, which will be referred to as haplotypes $1 \rightarrow 3$.

\section{Family study}

The first study to be reported consists of a series of parent-offspring comparisons with regard to atpA or coxI PCR/RFLP haplotype. If the inheritance of the mitochondrial genome is strictly maternal, and if all ovule donors are homoplasmic with regard to the mitochondrial genome, mother and offspring genotypes should be identical (barring mutation), regardless of the mitochondrial genotype of the pollen donor. With occasional paternal transmission, some offspring genotypes could match the father rather than the mother, or reflect a mixture of maternal and paternal genotypes (ie be heteroplasmic). Detection of heteroplasmy with PCRbased markers might depend on the ability of the PCR to amplify both versions of the gene simultaneously, which could be difficult if they are present in very unequal copy number. Undetected, or cryptic, heteroplasmy in one or both parents could be particularly problematic in family studies, because haplotypes too rare to be detected in a parent could still be transmitted to offspring, and then by chance increase in relative frequency during the successive episodes of replication and cell division associated with offspring growth. Thus, dissimilarity between an offspring and both of its parents could be an indication of heteroplasmy in one or both parents. 
The individuals used for this study were selected from a large number of sibships maintained in the Vanderbilt University greenhouse for other purposes (see Bailey and McCauley, 2005). The origin of the sibships is as follows. Hermaphroditic individuals collected as seeds from various North American populations were used as parents in one of two types of crosses, self-fertilization or outcrossing with another unrelated hermaphrodite. Seeds produced by these crosses (up to 50 seeds/cross) were planted in the greenhouse and raised to flowering. Following the crosses, the parental plants were discarded, but only after a DNA sample was taken from each parent, or a full sibling of that parent, using DNA extraction methods described above.

The PCR/RFLP atpA genotype of each parental line was determined by methods described above. Using this information, 23 groups of offspring were selected for study. Included were four sibships resulting from selffertilization, five sibships resulting from an outcross between two hermaphrodites carrying the same atp $A$ haplotype, and 14 sibships resulting from crosses between two hermaphrodites carrying different atp $A$ haplotypes. DNA was extracted from 10 to 15 members of each of the 23 sibships and used as a template in the PCR reaction necessary for determining the atp $A$ haplotype of that individual. Thus, the final data set allowed for 23 comparisons of the atp $A$ haplotypes of 10-15 full siblings with each other and with that of the maternal and paternal lines that created them.

A similar tactic was taken with regard to the coxI PCR/ RFLP haplotypes for the members of one family group showing strong evidence for paternal transmission (see below).

\section{Intraindividual study}

The possibility has been raised above that heteroplasmy might not be detected by a PCR-based marker if copies of one form of that marker are numerically dominant in the sample of DNA used as a template in the amplification reaction. In an effort to develop a method of detecting intraindividual variation in our mitochondrial markers that is otherwise cryptic to our standard protocol, the following technique was devised. Inspection of the original $\operatorname{atp} A$ sequence data revealed that $\operatorname{atp} A$ haplotypes C, D, E, and F share a single SmaI restriction site, whereas no SmaI sites are found in haplotype A or B sequences. Thus, digestion of the genomic DNA of individuals known to carry the C, D, E, or $\mathrm{F}$ atpA haplotype with SmaI should inhibit subsequent amplification of that DNA with the $\operatorname{atpA}$ primers. However, if the mitochondrial genome also carried a relatively few copies of the A or B forms of $\operatorname{atpA}$, these should still be suitable for amplification, and perhaps would amplify more efficiently under these conditions if the SmaI treatment knocked down any copies of the C, D, E, or $F$ forms that would otherwise be competitors during the reaction. Thus, the SmaI knockdown treatment might reveal cryptic heteroplasmy in individuals in which a minority of $\mathrm{A}$ and/or $\mathrm{B} \operatorname{atp} A$ molecules coexist with a majority of $\mathrm{C}, \mathrm{D}, \mathrm{E}$, or $\mathrm{F}$ molecules.

This approach was applied to 99 offspring individuals selected from the Family Study (and to their respective parental lines) using the following protocol. For each individual a $10 \mu \mathrm{l}$ aliquot of genomic DNA, taken from the same DNA extraction used as a template in the Family Study, was digested with SmaI. The digested DNA was then used as a template in the standard atpA PCR amplification. As a control, an additional $10 \mu \mathrm{l}$ aliquot of genomic DNA was subjected to the SmaI digestion protocol, except that water was substituted for the restriction enzyme. This was also used as a template in a separate PCR using the atp $A$ primers. The expectation is that the control reactions should generate the same $\operatorname{atp} A$ haplotype scored for that individual in the Family Study. For those individuals whose original haplotype was scored as C, D, E, or F, the SmaI knockdown treatment should yield either haplotype $\mathrm{A}$ or B, if that individual carried copies of those molecules at a low level, or no product if it was homoplasmic for C, $\mathrm{D}, \mathrm{E}$, or $\mathrm{F}$ molecules. Those parents whose original genotype was A or B should not be affected by the SmaI treatment.

\section{Evidence for recombination}

The presence of direct repeats within the plant mitochondrial genome facilitates the occurrence of intra- and intermolecular recombination events (Mackenzie and McIntosh, 1999). However, recombination within or between loci would be most likely to generate novel mitochondrial genotypes if intraindividual variation occurred at the locus or loci in question. Thus, evidence for intra- or interlocus recombination within the mitochondrial genome could also be indirect evidence for heteroplasmy (Städler and Delph, 2002).

Evidence for past intralocus recombination can be obtained from DNA sequences by examining the pattern of shared nucleotide polymorphism and conducting statistical tests designed to test the null hypothesis that patterns indicative of recombination are, in fact, due to homoplasy (Städler and Delph, 2002). To that end, atpA sequences representing one of each of the six $S$. vulgaris PCR/RFLP haplotypes were aligned manually and examined for shared nucleotide polymorphism. In addition, the $\operatorname{atp} A$ sequence obtained from one S. latifolia individual was used as an out-group.

At the population level, recombination between two polymorphic loci can be inferred from the presence of all possible two-locus genotypes (Saville et al, 1998). In order to evaluate the diversity of joint atpA/coxI haplotypes occurring in natural populations of $S$. vulgaris, seeds originating from a number of European populations of $S$. vulgaris were planted in the Vanderbilt University greenhouse and raised until enough foliage was produced for DNA extraction. Aliquots of this genomic DNA were used as a template for two PCR amplifications per individual, one using the $\operatorname{atp} A$ primers and one using the coxI primers. The resulting amplification products were used to determine an atp $A$ and a coxI PCR/RFLP haplotype for 89 individuals using methods outlined for the Family Study. Statistical independence of $\operatorname{atp} A$ and coxI haplotype identities across individuals was tested using a G-test of Independence (Sokal and Rohlf, 1995).

\section{Results}

\section{Family study}

The results of the crossing study are presented in Table 1. The $\operatorname{atp} A$ PCR/RFLP haplotype was determined for 318 
Table 1 atpA haplotypes of 23 full sib families created by greenhouse crosses of $S$. vulgaris lineages of known atpA haplotype

\begin{tabular}{rlll}
\hline Family & $\begin{array}{l}\text { Maternal } \\
\text { haplotype }\end{array}$ & $\begin{array}{l}\text { Paternal } \\
\text { haplotype }\end{array}$ & $\begin{array}{l}\text { \#Offspring/ } \\
\text { offspring } \\
\text { haplotype }\end{array}$ \\
\hline 1 & D & Self & $10 \mathrm{D}$ \\
2 & $\mathrm{~B}$ & Self & $10 \mathrm{~B}$ \\
3 & $\mathrm{~A}$ & Self & $10 \mathrm{~A}$ \\
4 & $\mathrm{C}$ & Self & $10 \mathrm{C}$ \\
5 & $\mathrm{~A}$ & $\mathrm{~A}$ & $15 \mathrm{~A}$ \\
6 & $\mathrm{E}$ & $\mathrm{E}$ & $15 \mathrm{E}$ \\
7 & $\mathrm{D}$ & $\mathrm{D}$ & $15 \mathrm{D}$ \\
8 & $\mathrm{D}$ & $\mathrm{D}$ & $14 \mathrm{D}, 1 \mathrm{~A}$ \\
9 & $\mathrm{D}$ & $\mathrm{D}$ & $15 \mathrm{D}$ \\
10 & $\mathrm{D}$ & $\mathrm{B}$ & $15 \mathrm{D}$ \\
11 & $\mathrm{~B}$ & $\mathrm{~A}$ & $15 \mathrm{~B}$ \\
12 & $\mathrm{~B}$ & $\mathrm{~A}$ & $15 \mathrm{~B}$ \\
13 & $\mathrm{~A}$ & $\mathrm{D}$ & $14 \mathrm{~A}, 1 \mathrm{E}$ \\
14 & $\mathrm{~A}$ & $\mathrm{~B}$ & $15 \mathrm{~A}$ \\
15 & $\mathrm{E}$ & $\mathrm{C}$ & $15 \mathrm{E}$ \\
16 & $\mathrm{~B}$ & $\mathrm{C}$ & $6 \mathrm{~B}, 1 \mathrm{~B} / \mathrm{C}$ a \\
17 & $\mathrm{C}$ & $\mathrm{B}$ & $15 \mathrm{C}$ \\
18 & $\mathrm{C}$ & $\mathrm{E}$ & $15 \mathrm{C}$ \\
19 & $\mathrm{C}$ & $\mathrm{A}$ & $15 \mathrm{C}$ \\
20 & $\mathrm{~B}$ & $\mathrm{~A}$ & $14 \mathrm{~B}$ \\
21 & $\mathrm{~A}$ & $\mathrm{~B}$ & $14 \mathrm{~A}, 1 \mathrm{C}$ \\
22 & $\mathrm{D}$ & $\mathrm{C}$ & $12 \mathrm{D}$ \\
23 & $\mathrm{D}$ & $\mathrm{A}$ &
\end{tabular}

a $\mathrm{B} / \mathrm{C}$ refers to an individual whose restriction pattern resembles an overlay of the B and C haplotypes.

offspring distributed among 23 families. Of these, 306 individuals $(96 \%)$ carried the expected maternal haplotype. Strict maternal inheritance was shown for at least one family carrying each of the five atpA haplotypes $(\mathrm{A} \rightarrow \mathrm{E})$ used in the study (no representatives of haplotype $\mathrm{F}$ were discovered), demonstrating that the results were not haplotype dependent. Four of the 23 sibships $(17 \%)$ contain at least one individual whose atpA genotype does not resemble the mother (see Figure 1 for an example), including three sibships in which the offspring genotype does not resemble either the maternal or the paternal line. In the fourth of these sibships (family 16), eight of 15 offspring resemble the paternal, rather than the maternal line. This group also contains an individual whose atpA restriction profile resembles what would be expected if the maternal and paternal profiles were superimposed over one another, much as would be expected in a heteroplasmic individual in which the maternal and paternal contributions were each amplified. The coxI PCR/RFLP genotype was also determined for every individual in family 16. The paternal and maternal lines used to create the family 16 sibship also carried different coxI PCR/RFLP haplotypes. Only the eight individuals that carried the paternal atp $A$ haplotype in family 16 also carried the paternal coxI haplotype.

\section{Intraindividual study}

The SmaI restriction knockdown method, as well as the control treatment, was applied to the genomic DNA of 99 offspring and their parents, distributed among 15 families. The results are summarized in Table 2. The control treatment of the parents and offspring yielded

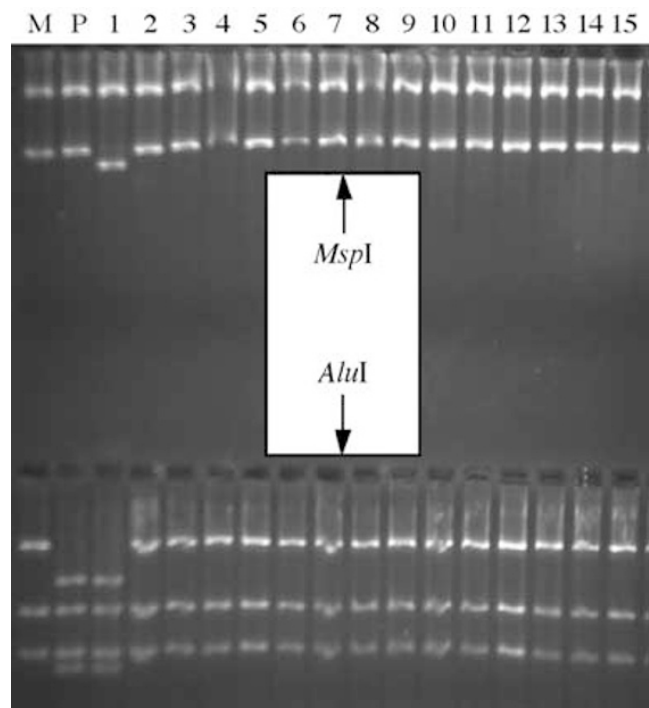

Figure $1 \mathrm{MspI}$ and $A l u \mathrm{I}$ restriction fragments observed following digestion of atpA PCR product obtained from maternal (M) or paternal (P) S. vulgaris individuals, along with 15 of their offspring. Note that offspring 2-15 carry haplotypes that match the maternal line (haplotype A), whereas offspring 1 carries a haplotype (C) that is not carried by either parent. The father is haplotype B.

Table 2 atp $A$ haplotype revealed when $S$. vulgaris genomic DNA is digested with SmaI prior to PCR with atpA primers

\begin{tabular}{lcl}
\hline Family/(cross) & $\begin{array}{c}\text { Original } \\
\text { haplotype }\end{array}$ & $\begin{array}{l}\text { Haplotype after } \\
\text { Smal treatment }\end{array}$ \\
\hline $1(\mathrm{D}$ self $)$ & $6 \mathrm{D}$ & $6 \mathrm{blank}$ \\
$4(\mathrm{C}$ self $)$ & $6 \mathrm{C}$ & 6 blank \\
$6(\mathrm{E} \times \mathrm{E})$ & $8 \mathrm{E}$ & 8 blank \\
$7(\mathrm{D} \times \mathrm{D})$ & $8 \mathrm{D}$ & 8 blank \\
$8\left(\mathrm{D} \times \mathrm{D}^{\mathrm{d}}\right)$ & $7 \mathrm{D}$ & $7 \mathrm{~A}$ \\
$10(\mathrm{D} \times \mathrm{B})$ & $8 \mathrm{D}$ & $8 \mathrm{blank}$ \\
$13(\mathrm{~A} \times \mathrm{D})$ & $1 \mathrm{E}$ & $1 \mathrm{~A}^{\mathrm{a}}$ \\
$15(\mathrm{E} \times \mathrm{C})$ & $8 \mathrm{E}$ & 8 blank \\
$16(\mathrm{~B} \times \mathrm{C})$ & $4 \mathrm{C}$ & $2 \mathrm{~B}^{\mathrm{a}}, 2$ blank \\
$17(\mathrm{C} \times \mathrm{B})$ & $8 \mathrm{C}$ & $7 \mathrm{~A}^{\mathrm{c}}, 1$ blank \\
$18(\mathrm{C} \times \mathrm{E})$ & $8 \mathrm{C}$ & 8 blank \\
$19(\mathrm{C} \times \mathrm{A})$ & $8 \mathrm{C}$ & $4 \mathrm{~A}^{\mathrm{b}}, 4 \mathrm{blank}$ \\
$21(\mathrm{~A} \times \mathrm{B})$ & $1 \mathrm{C}$ & $1 \mathrm{~A}^{\mathrm{a}}$ \\
$22(\mathrm{D} \times \mathrm{C})$ & $10 \mathrm{D}$ & $9 \mathrm{blank}, 1 \mathrm{~A}$ \\
$23(\mathrm{D} \times \mathrm{A})$ & $8 \mathrm{D}$ & $8 \mathrm{blank}$ \\
\hline
\end{tabular}

The original haplotype column presents the number of individuals from each sibship that were studied and their respective haplotypes in the Family Study. Blank refers to no PCR product after SmaI treatment. Superscripts a, b, and c refer to haplotypes that resemble the maternal haplotype, the paternal haplotype, and neither, respectively. Superscript $\mathrm{d}$ indicates that SmaI treatment of the paternal line also revealed haplotype A.

the same $\operatorname{atp} A$ haplotype seen for that individual in the Family Study. The SmaI treatment yielded the same atpA haplotype seen in the Family Study in the eight parents whose haplotype was A or B. The SmaI treatment resulted in a different atp $A$ haplotype in one paternal line of the remaining 20 parents, and in 23 of the 99 offspring (see Figure 2 for an example). In the remaining individuals, the SmaI-treated DNA template did not yield any PCR product. 

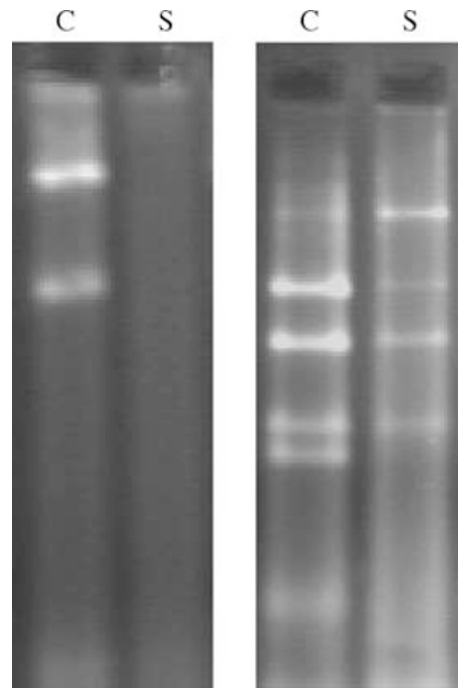

Figure $2 A l u \mathrm{I}$ restriction fragments observed for two $S$. vulgaris individuals when an $S m a I$ digestion treatment (S) or a control treatment (C) was applied to genomic DNA prior to PCR amplification using atpA primers. For the individual on the left, haplotype $\mathrm{E}$ is seen in the control, whereas no PCR product was obtained in the SmaI treatment. For the individual on the right, haplotype $\mathrm{C}$ is replaced by haplotype A following the SmaI treatment, an example of cryptic heteroplasmy.

In four of the offspring whose haplotype did not resemble the mother in the Family Study, treatment with SmaI prior to PCR yielded the maternal type. This included two individuals from Family 16 whose Family Study haplotype resembled the father. In seven individuals from Family 17, the SmaI haplotype does not match either parental line, whereas the Family Study haplotypes indicate maternal inheritance. In the one family in which the SmaI treatment differed from the control in the paternal line (Family 8), this same difference between treatments was also seen in seven of his offspring. It should be noted that the haplotype seen after SmaI treatment of this family (haplotype A) was identical to the haplotype seen in one member of this sibship that did not resemble the mother in the Family Study.

\section{Evidence for recombination}

In all, 11 shared polymorphic nucleotide sites were found within the S. vulgaris atpA sequences (Table 3 ). One observation of such data that is expected with recombination would be the occurrence of all recombinatorial pairs of nucleotides at some paired polymorphic positions, as is the case at paired positions $3-4$ (AG, AC, CG, and CC) and 8-9 (AA, AG, CA, and CG) (Table 3). There are a number of published statistical tests that can be used to evaluate evidence for recombination of this sort against a null model in which the apparent recombination is due to homoplasy. Each of these tests requires particular assumptions about the model of evolution and the sampling strategy (eg Saville et al, 1998; Städler and Delph, 2002). Inspection of Table 3 reveals a striking pattern that allows for a very simple statistical test conservative to these assumptions. The first four nucleotide polymorphisms found in haplotype
Table 3 Shared polymorphic nucleotides occurring within an $850 \mathrm{bp}$ region of the mitochondrial atp $A$ gene sequenced in one $S$. latifolia individual and six $S$. vulgaris individuals previously distinguished by atpA PCR/RFLP's

\begin{tabular}{|c|c|c|c|c|c|c|c|c|c|c|c|}
\hline \multirow[t]{2}{*}{ Haplotype } & \multicolumn{11}{|c|}{ Polymorphic nucleotides (relative position) } \\
\hline & 1 & 2 & 3 & 4 & 5 & 6 & 7 & 8 & 9 & 10 & 11 \\
\hline S. latifolia & $\mathrm{T}$ & $\mathrm{T}$ & A & A & G & $\mathrm{T}$ & G & A & A & $\mathrm{T}$ & A \\
\hline A & $\mathbf{T}$ & $\mathrm{T}$ & A & A & G & $\mathrm{T}$ & G & A & A & $\mathrm{T}$ & A \\
\hline C & $\overline{\mathbf{T}}$ & $\overline{\mathbf{T}}$ & $\overline{\mathbf{A}}$ & $\overline{\mathbf{A}}$ & C & G & A & $\mathbf{A}$ & G & $\mathbf{T}$ & G \\
\hline F & $\overline{\mathrm{A}}$ & $\overline{\mathrm{C}}$ & $\bar{G}$ & $\bar{C}$ & $\overline{\mathrm{C}}$ & $\overline{\mathrm{G}}$ & $\overline{\mathbf{A}}$ & $\overline{\mathbf{A}}$ & $\overline{\mathrm{G}}$ & $\overline{\mathbf{T}}$ & $\overline{\mathrm{G}}$ \\
\hline B & $\mathrm{T}$ & $\mathrm{T}$ & A & C & $\overline{\mathrm{G}}$ & $\overline{\mathrm{T}}$ & $\overline{\mathrm{G}}$ & $\overline{\mathrm{C}}$ & $\overline{\mathrm{A}}$ & $\overline{\mathrm{T}}$ & $\overline{\mathrm{A}}$ \\
\hline $\mathrm{D}$ and $\mathrm{E}$ & A & C & G & C & C & G & A & C & G & C & A \\
\hline
\end{tabular}

Table 4 Number of joint atpA/coxI haplotypes found in a sample of 89 S. vulgaris individuals

\begin{tabular}{lcrc}
\hline & \multicolumn{3}{c}{ CoxI haplotype } \\
\cline { 2 - 4 } & \multicolumn{1}{c}{1} & 2 & 3 \\
\hline atpA haplotype & & & \\
A & $24(30.2)$ & $28(24.0)$ & $9(6.9)$ \\
B & $20(13.8)$ & $7(11.0)$ & $1(3.1)$ \\
\hline
\end{tabular}

Expected values under a null hypothesis of random association of haplotypes between loci are given in parenthesis.

Note: Several individuals with rare atpA haplotypes are not included.

C are shared with haplotype A and S. latifolia, whereas the last seven polymorphisms found in haplotype $C$ are shared with haplotype F. This pattern could be viewed as evidence for either intragenic recombination or homoplasy. A simple statistical test to distinguish these two possibilities first assumes that haplotype $C$ is derived from haplotype $\mathrm{F}$ without recombination, and that the TTAA motif it shares with haplotype A is due to homoplasy. Assuming that each of the four nucleotide positions has undergone a mutation, and that all nucleotide-nucleotide transitions are equally likely, yields a probability of a ACGC $\rightarrow$ TTAA transition of $(1 / 3)^{4}$ or 0.012 . (Assuming that haplotype $\mathrm{F}$ is derived from haplotype A without recombination yields a probability of a GTGAATA $\rightarrow$ CGAAGTG transition of $(1 / 3)^{5}$ or 0.004 .) Although more realistic assumptions incorporating a much lower mutation rate and unequal nucleotide usage would undoubtedly yield a lower probability of homoplasy, the simple conservative test used here provides strong statistical evidence for a recombination event.

The joint atpA/coxI haplotype was determined for $89 \mathrm{~S}$. vulgaris individuals collected from natural populations in Europe. Several additional individuals carrying rare atp $A$ haplotypes not detected previously were excluded from the analysis. Note that atpA haplotypes C, D, E, and F were not found in the European sample. The distribution of two-locus genotypes is presented in Table 4. It can be seen that one or more individuals carrying each of the possible two-locus genotypes were found. However, the two loci are not in gametic equilibrium, in that alleles at the two loci are not in random association with one another across individuals $\left(G_{\text {independence }}=8.69, \mathrm{df}=2\right.$, $P=0.013)$. 


\section{Discussion}

The main results of the studies of $S$. vulgaris presented here are: (1) mother and offspring mtDNA haplotypes do not always match when PCR products derived from coding regions are used as mitochondrial genetic markers in controlled crosses, (2) more than one form of these mitochondrial genetic markers can coexist within the same individual, and (3) there is evidence of both intra- and interlocus recombination. These results are consistent with the hypotheses that (1) the inheritance of the mitochondrial genome in S. vulgaris is not strictly maternal, (2) an individual can inherit copies of the mitochondrial genome from both parents simultaneously, resulting in a heteroplasmic state, and (3) heteroplasmy facilitates the creation of novel mitochondrial genotypes through intra- and intergenic recombination. It would be useful to consider how more specific details of the results support these hypotheses, how the results might be interpreted in light of competing alternate hypotheses, and how the results presented here compare to results from previous studies of $S$. vulgaris.

When considering evidence for transmission of the mitochondrial genome through pollen, one should probably assume that copies of the mitochondrial genome are likely to be transmitted to the embryo through the ovule as well. Further, it is possible that the relative frequencies of the maternally and paternally derived copies could change by chance during growth and development (Birky, 2001; Rand, 2001). Documentation of paternal transmission in the Family Study would occur when copies of the mitochondrial genome transmitted through pollen occur at a number sufficient to be detectable by PCR, perhaps by increasing in relative frequency by intraindividual drift during growth. Should that happen, and if the maternal line of molecules is lost by chance, or remains at a level not easily detected by PCR, the inheritance would appear to be paternal. The best evidence for paternal transmission of this sort comes from family 16, in which eight of 15 offspring match the paternal, rather than the maternal line, for both the atp $A$ and coxI markers.

Retention of both paternally derived and maternally derived copies of the mitochondrial genome at relatively high copy number during development would result in a heteroplasmic individual whose PCR/RFLP profile resembles an overlay of the two parental types. This was seen in one member of the family 16 sibship in the Family Study. With biparental inheritance, there must also be cases in which a paternal lineage of molecules is transmitted to the embryo during fertilization but does not rise to a frequency high enough to dominate the PCR. This hypothesis suggests that there should exist a number of cryptically heteroplasmic individuals in which either the maternal or paternal lineage of molecules exists at levels that are difficult to detect by PCR, when in competition with a majority lineage traced to the other parent. Support for this possibility comes from the knockdown experiment in which treatment with SmaI caused two of the members of family 16 displaying paternal inheritance in the Family Study to display the maternal genotype, and several members of family 19 displaying maternal inheritance in the Family Study to display the paternal genotype.
Given that an individual is heteroplasmic, a heterogeneous collection of molecules could be passed to its offspring, even when inheritance is uniparental. A haplotype that is at low frequency in a cryptically heteroplasmic parent could, by chance, drift to high frequency during maturation of a minority of its offspring, resulting in the occasional appearance of an unexpected genotype in a PCR survey. Remember that two of the individuals that did not resemble either parent in the Family Study did display the maternal haplotype after SmaI treatment. In addition, cases of father-offspring haplotype matches were revealed in family 8 after the SmaI treatment revealed the father to be cryptically heteroplasmic.

An alternate hypothesis that must be considered is that there could exist two or more paralogous copies of atpA in the mitochondrial genome of $S$. vulgaris that can be amplified by the PCR primers used in this study. Perhaps one or the other of the paralogs amplifies differentially, depending on the family being studied. One reason for the differential PCR amplification might be large differences between paralogs in copy number. Studies of the mitochondrial genomes of several plant species have documented a phenomenon known as substoichiometric shifting in which subgenomic mtDNA molecules, normally maintained in low abundance relative to the rest of the mitochondrial genome, can shift in copy number abruptly between generations (Mackenzie and McIntosh, 1999). This process is most likely under nuclear control and has been implicated in CMS in some species (Mackenzie and McIntosh, 1999; Arrieta-Montiel et al, 2001). If paralogous copies of $\operatorname{atp} A$ are involved in such a process, shifting events could result in dissimilarity between mother and offspring in the atpA haplotype scored by PCR, of the sort seen in the Family Study. The cryptic heteroplasmy revealed by the SmaI knockdown experiment could be a consequence of the effects of the inequity in copy number on the outcome of PCR. The key difference between the paternal transmission hypothesis and the substoichiometric shifting hypothesis is that, in the latter, mother-offspring dissimilarity and apparent heteroplasmy could be observed even if the mitochondrial genome is strictly maternally inherited. A secondary difference is that under the shifting hypothesis deterministic forces, rather than intraindividual genetic drift, determine relative copy number.

While paternal transmission and substoichiometric shifting would be expected to generate several similar empirical observations, given the experiments described here, some specific features of the data set seem best explained by the paternal transmission hypothesis. Foremost is the observation that eight members of family 16 carry the paternal haplotype for both $\operatorname{coxI}$ and atpA. Under the substoichiometric shifting hypothesis, one would have to imagine that there were paralogous copies of both loci whose copy number shifted simultaneously, and that the observed father-offspring similarity was coincidental for both loci. In addition, there are a number of examples in the Intraindividual Study in which SmaI treatment of offspring DNA exposed a cryptic atpA haplotype also seen in the paternal, but not the maternal line.

The results of several previous studies of the genus Silene are relevant to the interpretation of those presented 
here. Two other studies of $S$. vulgaris have used genetic markers to investigate the inheritance of the mitochondrial genome in this species (Olson and McCauley, 2000; Andersson-Ceplitis and Bengtsson, 2002). Strict maternal inheritance was found in both cases. However, given the large sample sizes needed to detect rare paternal inheritance with a high degree of probability (Milligan, 1992), we do not regard the low rate of nonmaternal inheritance reported here and the results of the previous studies as necessarily contradictory. Milligan (1992) presents an equation based on the binomial distribution that is often used to set an upper bound on the probability of nonmaternal inheritance, given a sample size $(n)$ and an arbitrary degree of certainty. For example, Olson and McCauley (2000) state that given their results there is a $95 \%$ probability that the actual rate of nonmaternal inheritance is less than $19.3 \%$. Thus, while they showed convincingly that the inheritance of the mitochondrial genome is predominantly maternal, their power to detect rare nonmaternal inheritance events at a frequency comparable to that reported here was relatively weak. Given their much larger sample sizes, Andersson-Ceplitis and Bengtsson (2002) had the statistical power to detect nonmaternal inheritance with $95 \%$ probability if its true rate of occurrence is greater than about $1 \%$. However, the equation suggested by Milligan (1992) assumes that each observation is independent, which may not always be the case. Typically, the Milligan calculations are based on a sample size $n$ that is obtained by pooling some number of offspring from each of a relatively small number of crosses (as is the case in Olson and McCauley 2000; Andersson-Ceplitis and Bengtsson, 2002 and this study). There is evidence from studies of chloroplast DNA (cpDNA) (Birky, 1995; Mogensen, 1996) that the probability of nonmaternal inheritance can vary among crosses or among genotypes. If this is the case, the statistical power to detect nonmaternal inheritance decreases as the total number of observations is apportioned among fewer and fewer crosses, since observations within families are not independent. Thus, the results presented here demonstrate that nonmaternal inheritance can occur upon occasion, but do not allow for estimation of its rate with any degree of precision, whereas the previous studies demonstrated that nonmaternal inheritance is rare, but do not eliminate the possibility that it could occur at a frequency comparable to that reported here.

Evidence for mitochondrial heteroplasmy in S. vulgaris was presented in a study by Andersson (1999) based on differences in the offspring sex ratios produced by female and hermaphrodite flowers found on the same gynomonoecious individual, and on the spatial distribution of the two flower morphs across the plant. A plausible interpretation of these results is that gynomonoecious individuals inherit more than one form of CMS, and that these forms segregate spatially within the plant during growth and development (Andersson, 1999). In a study of $S$. acaulis, a related gynodioecious species, Städler and Delph (2002) suggested that their evidence for mitochondrial intragenic recombination implied the existence of heteroplasmy.

Two previous observations of the population genetics of $S$. vulgaris would not be expected, given the results presented here. A perfect association between mitochondrial nad4 restriction haplotype and the presence or absence of mitochondrial plasmids was found in a study of Swedish populations of $S$. vulgaris by AnderssonCeplitis (2002). Occasional paternal transmission or heteroplasmy in the mitochondrial genome would be expected to break up these associations. Olson and McCauley (2000) studied the joint distribution of cpDNA and mtDNA haplotypes among $S$. vulgaris individuals collected from a number of local populations in southwestern Virginia. They found a perfect association between cpDNA and mtDNA haplotypes across individuals. No mtDNA haplotype was associated with more than one cpDNA haplotype. Multiple mtDNA haplotypes were nested within some cpDNA types. This is what one might expect if cpDNA and mtDNA were both strictly maternally inherited, but evolved at different rates. However, a recent study of $\mathrm{cpDNA}$ and mtDNA variation in $S$. vulgaris populations in Europe by Storchova and Olson (2004) found cases of mtDNA haplotypes nested within cpDNA types and other cases in which cpDNA haplotypes were nested within mtDNA types.

It remains to be seen how the paternal transmission and heteroplasmy documented here by greenhouse crosses influence the genetics of natural populations. Several features of the population biology of $S$. vulgaris would seem to reduce the opportunity for paternal transmission to impact the population genetics of the mitochondrial genome. Most importantly, a study of $S$. vulgaris populations in Virginia by Emery (2001) has shown that hermaphrodites have a self-fertilization rate of about $40 \%$. Paternal transmission with self-fertilization would not introduce additional heterogeneity to a mitochondrial genome. Similarly, mitochondrial genes display modest to high local population structure (Andersson-Ceplitis, 2002; Olson and McCauley, 2002; Storchova and Olson, 2004), and so a significant fraction of outcross events would be between genetically similar individuals. Recall, however, that one case of cryptic heteroplasmy was discovered in a paternal individual that was collected as a seed from a natural population. Further, the evidence for mitochondrial recombination in our study of European populations suggests the signature of biparental inheritance and heteroplasmy, as does the evidence for heteroplasmy presented by Andersson (1999). Further studies of natural populations are clearly indicated.

The evidence presented here for paternal transmission and heteroplasmy in the mitochondrial genome of $S$. vulgaris comes from studies of two mitochondrial genes not known to be involved in CMS in this species. The relevance of these results for the study of gynodioecy depends on the likelihood that mitochondrial CMS elements behave similarly. Until the CMS elements are identified molecularly, it will be difficult to study their inheritance directly. Still, the possibility of occasional paternal transmission of mitochondrial CMS elements, and that this paternal transmission can result in heteroplasmy, is intriguing. When transmission of CMS is not strictly maternal, any advantage enjoyed by females in seed production does not translate directly into a fitness advantage for the cytoplasmic genes that she carries, perhaps influencing the dynamics of sex ratio evolution. How heteroplasmy for CMS affects sex expression is unclear, but must depend, in part, on how the different CMS factors become distributed spatially across the 
plant. Full restoration of a plant heteroplasmic for CMS would be difficult if it requires distinct restorer alleles for each type of CMS carried by that plant. Given the results presented here for S. vulgaris, it would seem important to consider the possibility of nonmaternal inheritance and heteroplasmy when studying the population biology of any gynodioecious plant species for which cytonuclear sex determination is suspected, but in which the inheritance of the mitochondrial genome is not known.

\section{Acknowledgements}

We thank Claude $\mathrm{W}$ dePamphilis for providing the atp $A$ and coxI primer sequences used to generate the RFLP markers, Jonathan Ertelt for his expertise and assistance in the greenhouse, and Alan Smith, David Wills, and Mark Welch for their efforts in the lab. Seeds collected from European S. vulgaris populations were provided by Helene Andersson-Ceplitis, Melanie Glaettli, Mathew Olson, Helena Storchova, and Douglas Taylor. Conversations with John Burke, Matt Olson, Douglas Taylor, and Michael Wade greatly clarified our thinking concerning the potential theoretical consequences of the results presented here. Financial support was provided by a grant from the National Science Foundation to DEM (DEB-0078531) and a Vanderbilt University Discovery Grant.

\section{References}

Andersson H (1999). Female and hermaphrodite flowers on a chimeric gynomonoecious Silene vulgaris plant produce offspring with different genders: a case of heteroplasmic sex-determination? J Hered 90: 563-565.

Andersson-Ceplitis H (2002). Evolutionary dynamics of mitochondrial plasmids in natural populations of Silene vulgaris. Evolution 56: 1592-1598.

Andersson-Ceplitis H, Bengtsson BO (2002). Transmission rates and phenotypic effects of mitochondrial plasmids and cytotypes in Silene vulgaris. Evolution 56: 1586-1591.

Arrieta-Montiel M, Lyznik A, Wolosznska M, Janska H, Tohme J, Mackenzie S (2001). Tracing evolutionary and developmental implications of mitochondrial stoichiometric shifting in the common bean. Genetics 158: 851-864.

Bailey MF, McCauley DE (2005). Offspring sex ratio under inbreeding and outbreeding in a gynodioecious plant. Evolution 59: 287-295.

Barkman TJ, Chenery G, McNeal JR, Lyons-Weiler J, Elisens WJ, Moore G et al (2000). Independent and combined analyses of sequences from all three genomic compartments converge on the root of flowering plant phylogeny. Proc Natl Acad Sci USA 97: 13166-13171.

Birky CW (1995). Uniparental inheritance of mitochondrial and chloroplast genes: mechanisms and evolution. Proc Natl Acad Sci USA 92: 11331-11338.

Birky CW (2001). The inheritance of genes in mitochondria and chloroplasts: laws, mechanisms and models. Annu Rev Genet 35: 125-148.

Bowe LM, Coat G, dePamphilis CW (2000). Phylogeny of seed plants based on all three genomic compartments: extant gymnosperms are monophyletic and Gnetales' closest relatives are conifers. Proc Natl Acad Sci USA 97: 4092-4097.

Budar F, Touzet P, De Paepe R (2003). The nucleo-mitochondrial conflict in cytoplasmic male sterilities revisited. Genetica 117: 3-16.

Charlesworth D, Laporte V (1998). The male-sterility polymorphism of Silene vulgaris: analysis of genetic data from two populations and comparison with Thymus vulgaris. Genetics 150: $1267-1282$.

Cosmides LM, Tooby J (1981). Cytoplasmic inheritance and intragenomic conflict. Theor Biol 89: 83-129.

Couvet D, Atlan A, Belhassen E, Gliddon C, Gouyon PH, Kjellberg F (1990). Co-evolution between two symbionts: the case of cytoplasmic male-sterility in higher plants. Oxford Surv Evol Biol 7: 225-250.

Doyle JJ, Doyle JL (1987). A rapid DNA isolation procedure for small amounts of fresh leaf tissue. Phytochem Bull 19: 11-15.

Emery SN (2001). Inbreeding depression and its consequences in Silene vulgaris. Masters Thesis, Vanderbilt University, TN, USA.

Emery SN, McCauley DE (2002). Consequences of inbreeding for offspring fitness and gender in Silene vulgaris, a gynodioecious plant. J Evol Biol 15: 1057-1066.

Frank SA (1989). The evolutionary dynamics of cytoplasmic male sterility. Am Nat 133: 345-376.

Hurst LD, Atlan A, Bengtsson BO (1996). Genetic conflicts. Quart Rev Biol 71: 317-364.

Ingvarsson PK, Taylor DR (2002). Geneological evidence for epidemics of selfish genes. Proc Natl Acad Sci USA 99: 11265-11269.

Jacobs MS, Wade MJ (2003). A synthetic review of gynodioecy. Am Nat 161: 837-851.

Jolls CL, Chenier TC (1989). Gynodioecy in Silene vulgaris (Caryophyllaceae): progeny success, experimental design and maternal effects. Am J Botany 76: 1360-1367.

Mackenzie S, McIntosh L (1999). Higher plant mitochondria. Plant Cell 11: 571-585.

McCauley DE (1998). The genetic structure of a gynodioecious plant: nuclear and cytoplasmic genes. Evolution 52: 255-260.

McCauley DE, Brock MT (1998). Frequency-dependent fitness in Silene vulgaris, a gynodioecious plant. Evolution 52: 30-36.

McCauley DE, Olson MS, Emery SN, Taylor DR (2000). Population structure influences sex ratio evolution in a gynodioecious plant. Am Nat 155: 814-819.

Milligan BG (1992). Is organelle DNA strictly maternally inherited? Power analysis of a binomial distribution. Am Bot 79: 1325-1328.

Mogensen HL (1996). The hows and whys of cytoplasmic inheritance in seed plants. Am J Bot 83: 383-404.

Nagata N, Saito C, Sakai A, Kuroiwa H, Kuroiwa T (1999). The selective increase or decrease of organellar DNA in generative cells just after pollen mitosis one controls cytoplasmic inheritance. Planta 209: 53-65.

Olson MS, McCauley DE (2000). Linkage disequilibrium and phylogenetic congruence between chloroplast and mitochondrial haplotypes in Silene vulgaris. Proc $R$ Soc Lond Ser B 267: 1801-1808.

Olson MS, McCauley DE (2002). Mitochondrial DNA diversity, population structure, and gender association in the gynodioecious plant Silene vulgaris. Evolution 56: 253-262.

Pettersson MW (1992). Advantages of being a specialist female in gynodioecious Silene vulgaris S.L. (Caryophyllaceae). Am J Bot 79: 1389-1395.

Rand DM (2001). The units of selection on mitochondrial DNA Annu Rev Ecol Syst 32: 415-448.

Reboud X, Zeyl C (1994). Organelle inheritance in plants. Heredity 72: 132-140.

Saumitou-Laprade P, Cugen J, Vernet P (1994). Cytoplasmic male sterility in plants - molecular evidence and the nucleocytoplasmic conflict. Trends Ecol Evol 9: 431-435.

Saville BJ, Kohl Y, Anderson JB (1998). mtDNA recombination in a natural population. Proc Natl Acad Sci USA 95: 1331-1335.

Sokal RR, Rohlf FJ (1995). Biometry, 3rd edn. Freeman: New York.

Städler T, Delph LF (2002). Ancient mitochondrial haplotypes and evidence for intragenic recombination in a gynodioecious plant. Proc Nat Acad Sci USA 99: 11730-11735. 
Storchova H, Olson MS (2004). Comparison between mitochondrial and chloroplast DNA variation in the native range of Silene vulgaris. Mol Ecol 13: 2909-2913.

Taylor DR, Olson MS, McCauley DE (2001). A quantitative genetic analysis of nuclear-cytoplasmic male sterility in structured populations of Silene vulgaris. Genetics 158: 833-841.
Werren JH, Beukeboom LW (1998). Sex determination, sex ratios, and genetic conflict. Ann Rev Ecol Syst 29: 233-261.

Zhang Q, Liu Y, Sodmergen (2003). Examination of the cytoplasmic DNA in male reproductive cells to determine the potential for cytoplasmic inheritance in 295 angiosperm species. Plant Cell Physiol 44: 941-951. 